\section{Horner's syndrome in patients admitted to the intensive care unit that have undergone central venous catheterization: a prospective study}

Z Butty', J Gopwani' ${ }^{1,2}$, S Mehta ${ }^{3}$ and E Margolin ${ }^{1,3}$

\begin{abstract}
Purpose Central venous catheterization (CVC) is estimated to be performed in millions of patients per year. Swan-Ganz catheters used for CVC are most often inserted into the internal jugular vein and during this procedure they may come into contact with the sympathetic chain. This study aims to determine the incidence of Horner's syndrome in patients admitted to intensive care unit that have undergone internal jugular CVC insertion during their admission and to determine whether ultrasonography-assisted insertion has decreased the frequency of this complication. Patients and methods A total of 100 prospective patients admitted to the ICU were examined for the presence of anisocoria and ptosis after undergoing recent CVC. Presence of Horner's syndrome was confirmed by testing with $0.5 \%$ apraclonidine and looking for the reversal of anisocoria.

Results Frequency of Horner's syndrome after CVC was $2 \%$ in a sample of 100

per year. Mechanical, thrombotic, infectious, and neurologic complications associated with this procedure have been well documented. ${ }^{1,2}$ SwanGanz catheter used for CVC is most often inserted into the internal jugular vein to assist in intravascular volume resuscitation and during this procedure it may come into contact with the sympathetic chain. Isolated case studies of patients with Horner's syndrome post CVC have been documented in the past ${ }^{3-7}$ with one prospective study looking at the incidence of neurological complications after CVC insertion, ${ }^{2}$ however, the frequency of Horner's syndrome has not been documented since the introduction of ultrasonography-assisted insertion. 8,9

This study aims to determine the incidence of Horner's syndrome in patients admitted to intensive care unit that have undergone internal jugular CVC insertion during their admission and to determine whether ultrasonographyassisted insertion has decreased the frequency of this complication.
\end{abstract} prospectively examined patients.

Conclusion Horner's syndrome remains a relatively rare but definitive complication of CVC. ICU physicians should be educated about its existence and prevalence and ophthalmologists should inquire about any history of ICU admission necessitating CVC insertion in any patient presenting with Horner's syndrome.

Eye (2016) 30, 31-33; doi:10.1038/eye.2015.181; published online 18 September 2015

\section{Introduction}

Central venous catheterization $(\mathrm{CVC})$ is estimated to be performed in millions of patients

\section{Patients and methods}

The study was conducted at Mount Sinai Hospital intensive care unit, a tertiary medicalsurgical ICU affiliated with the University of Toronto, between July of 2013 and April of 2014 . The study was approved by the Mount Sinai Research Ethics Board (REB). Consent from the patient or substitute decision maker was obtained prior to enrollment. In this tertiary care ICU, all CVC insertions are routinely conducted with ultrasonographic real-time guidance.

One hundred (100) consecutive patients who had a central venous line inserted in the internal jugular vein by ICU personnel within the prior
${ }^{1}$ Department of Ophthalmology and Vision Sciences, University of Toronto, Toronto, Ontario, Canada

${ }^{2}$ Department of Ophthalmology, University of West Indies, Kingston, Jamaica

${ }^{3}$ Department of Medicine, University of Toronto, Toronto, Ontario, Canada

Correspondence: E Margolin, Department of Ophthalmology and Vision Sciences, University of Toronto, 801 Eglinton Ave West, Suite 301, Toronto, Ontario M5N 1E3, Canada Tel: +647 748 8377; Fax: +416 6195539 E-mail: edmargolin@ gmail.com

Received: 27 April 2015 Accepted in revised form: 20 July 2015

Published online: 18 September 2015 
5 days were enrolled. This sample size was chosen as it was practical and it was much higher than any previously studied sample described in the literature. ${ }^{2}$ Exclusion criteria included subjects who were known to have preexisting Horner's syndrome, subjects with suspicion of prior Horner's syndrome (who had ipsilateral ptosis in photographs prior to admission), subjects with a known allergy to apraclonidine eye drops, as well as subjects for whom informed consent was not obtained.

We collected data on operators' experience defined by their level of training (year of post graduate training for the residents or staff), number of attempts made to insert the catheter, whether ultrasonography-assisted insertion was used, and any complications associated with the insertion (mainly carotid artery puncture).

Within 5 days of CVC insertion, each subject had an ophthalmologic exam by one of the investigators (ZB: PGY 3; or JG: PGY 6). The examination involved a careful pupillary examination for the presence of anisocoria over $1 \mathrm{~mm}$ and the presence of ptosis on the side with the smaller pupil. If the examination findings were suspicious for Horner's syndrome, pharmacological testing was performed. Apraclonidine $0.5 \%$ drop was instilled in each eye and the subject was re-examined in 60 min looking for the reversal of anisocoria. Pupils were photographed with a digital camera pre and post apraclonidine instillation. Horner's syndrome was confirmed if there was at least $1 \mathrm{~mm}$ dilation of the smaller pupil. Confirmed cases of Horner's syndrome were further investigated with an MRI/MRA of the head and neck and chest x-ray.

\section{Results}

In all, 100 patients who had internal jugular CVC insertion during the study period were enrolled in the study. Mean age was 59.8 \pm 16.6 years (range 22 to 92 years); there were 64 men and 36 women.

Ultrasonography-assisted insertion was performed in 98 subjects (98\%). The level of training of the operators was as follows: PGY-1: 6\%; PGY-2: 49\%; PGY-3: 7\%; PGY-4: 8\%; Fellows: 25\%; PGY-2 and PGY-3 with staff: $2 \%$; and staff alone: $1 \%$. The mean number of attempts for the CVC insertion was 1.49 (range 1-6).

Anisocoria of $1 \mathrm{~mm}$ or more was found in $9(9 \%)$ patients and ptosis was found in 4 (4\%). Out of the 4 patients who had ptosis and anisocoria, 3 had anisocoria with a smaller pupil on the ipsilateral side of the IJ line insertion and 1 patient had anisocoria with the ptosis on the contralateral side. 5 patients had anisocoria with no ptosis and all tested negative with apraclonidine $0.5 \%$.

Two patients with ptosis on the same side as the IJ line insertion tested positive with $0.5 \%$ apraclonidine with reversal of anisocoria and thus diagnosed with Horner's syndrome. None of these patients had anisocoria or ptosis on the photographs from before their hospital admission. One patient who had ptosis and anisocoria on the same side as the IJ line did not have reversal of anisocoria with apraclonidine despite being tested twice on two different days and re-examined for the reversal of anisocoria $60 \mathrm{~min}$ and $3 \mathrm{~h}$ after each test.

Two patients had carotid puncture and four had local bleeding as a complication of insertion but all complications were successfully managed. None of these patients developed Horner's syndrome.

Two patients who developed Horner's syndrome had right-sided IJ insertion performed by PGY-2 level trainees with only one uncomplicated attempt of insertion in each patient. Both patients were investigated upon their discharge from the ICU and no other reasons for Horner's syndrome was found.

\section{Statistical analysis}

Incidence of Horner's syndrome in our population was $2 \%$. Fisher's exact test did not demonstrate any difference in Horner's syndrome incidence in anatomical vs ultrasound-guided insertion of CVC. We note however that the sample size and the incidence were low (2\%; 95\% CI, 0.34 to $0.74 \%)$.

\section{Discussion}

Horner's syndrome is an uncommon complication of CVC. Horner's syndrome classically manifests as ipsilateral blepharoptosis, pupillary miosis, and facial anhidrosis. The syndrome results from the insult to the oculosympathetic pathway which follows a long, circuitous route with central, preganglionic, and postganglionic neurons. Depending on the anatomic location of the underlying pathologic process, Horner's syndrome is classified into central, preganglionic, and postganglionic types. ${ }^{10-12}$

Horner's syndrome associated with CVC of the internal jugular vein is likely caused by the damage to the sympathetic plexus or peri-subclavian neural loops that are closely adjacent to the carotid sheath (first-order neurons in the sympathetic chain). Most likely it is a misdirected cannula causing the direct trauma to the sympathetic plexus or an expanding hematoma resulting from the cannula misdirection that produces Horner's syndrome post CVC of the internal jugular vein. 3,13

Garcia et $a l^{2}$ reported an incidence of Horner's syndrome $(2 \% ; 95 \%$ CI, 1 to $8 \%)$ in a prospective study of 66 patients who had internal jugular CVC insertion without ultrasound guidance. In that study Horner's syndrome was detected in only one patient and it was thought to be associated with the large carotid sheath hematoma that developed during the catheter insertion. 
In our study where the largest number of patients were prospectively examined in the ICU post-CVC placement we noted no correlation between the incidence of Horner's syndrome and the use of ultrasonographyassisted insertion. We conclude that Horner's syndrome remains a relatively rare but definitive complication of CVC even in the era of ultrasound-guided insertion and ICU physicians should be educated about its existence and prevalence. Similarly, we recommend that ophthalmologists should inquire about any history of ICU admission necessitating CVC insertion in any patient presenting with acute Horner's syndrome.

Efforts to minimize and prevent the occurrence of CVC complications should be a routine element of quality improvement programs. Although emphasis is placed on the growing body of information that supports the use of ultrasound-assisted insertion (UAI) as a superior technique to decrease adverse events from CVC insertion, ${ }^{14}$ the ultrasound guided insertion had similar rate of HS occurrence as previously reported anatomical insertion without ultrasound guidance. ${ }^{2}$

To our knowledge, this study has the largest prospective data in the literature investigating the incidence of Horner's syndrome post-CVC insertion. As a limitation of our study, however, we acknowledge that as the subjects were screened within 5 days of central catheter insertion, some patients could have developed clinical manifestations of Horner's syndrome after that time frame. It has been previously reported though that apraclonidine testing can become positive after sympathetic pathway trauma as early as $3 \mathrm{~h}$ after the insult. ${ }^{15}$ It is also worth mentioning that it can be difficult to determine subtle miosis and ptosis in the critically ill patients with reduced levels of consciousness and we could have missed patients with subtle clinical findings in our screening process.

\section{Summary}

What was known before

- Horner's syndrome is a known complication of central venous cathetherization.

- Exact incidence of Horner's syndrome post CVC is unknown after ultrasound-guided CVC was introduced.

What this study adds

- Incidence of Horner's syndrome after CVC.

- Incidence of Horner's syndrome post CVC is significant and should be considered in the differential.

\section{Conflict of interest}

The authors declare no conflict of interest.

\section{Acknowledgements}

We thank and acknowledge our research coordinator Brittany Giacomino, as well as all the patients that participated in our study.

\section{References}

1 McGee DC, Gould MK. Preventing complications of central venous catheterization. $N$ Engl J Med 2003; 348: 1123-1133.

2 Garcia E, Wijdicks EF, Younge BR. Neurologic complications associated with internal jugular vein cannulation in critically ill patients: a prospective study. Neurology 1994; 44: 951-952.

3 Suominen PK, Korhonen AM, Vaida SJ, Hiller AS. Horner's Syndrome secondary to internal jugular venous cannulation. J Clin Anesth 2008; 20(4): 304-306.

4 Knyazer B, Levy J, Rosenburg E, Lifshitz T, Lazer I. Horner's Syndrome in an infant with complicated pneumonia. Isr Med Assoc J 2011; 13(8): 504-506.

5 Jarvis J, Watson A, Roberson G. Horner's syndrome after central venous catheterization. N Z Med J 2005; 118(1215): U1470.

6 Alrubaiy L, Khan A. Horner's syndrome following internal jugular vein cannulation. Emerg Med J 2011; 28(4): 343.

7 Reddy G, Coombes A, Hubbard AD. Horner's syndrome following internal jugular vein cannulation. Intensive Care Med 1998; 24: 194-196.

8 Fry WR, Clagett GC, O'Rourke PT. Ultrasound-guided central venous access. Arch Surg 1999; 134(7): 738-740.

9 Gann Jr M, Sardi A. Improved results using ultrasound guidance for central venous access. Am Surg 2003; 69(12): 1104-1107.

10 Morales J, Brown SM, Abdul-Rahim AS, Crosson CE. Ocular effects of Apraclonidine in Horner syndrome. Arch Ophthalmol 2000; 118: 951-954.

11 Walton KA, Buono LM. Horner syndrome. Curr Opin Ophthalmol 2003; 14(6): 357-363.

12 Davagnanam I, Fraser CL, Miszkiel K, Daniel CS, Plant GT. Adult Horner's syndrome: a combined clinical, pharmacological, and imaging algorithm. Eye 2013; 27(3): 291-298.

13 Links DJ, Crowe PJ. Horner's Syndrome after placement of a peripherally inserted central catheter. JPEN J Parenter Enteral Nutr 2006; 30(5): 451-452.

14 Dilisio R, Mittnacht AJ. The 'medial-oblique' approach to ultrasound-guided central venous cannulatio-maximize the view, minimize the risk. J Cardiothorac Vasc Anesth 2012; 26(6): 982-984

15 Cooper-Knock J, Pepper I, Hodgson T, Sharrack B. Early diagnosis of Horner syndrome using topical apraclonidine. J Neuroophthalmol 2011; 31(3): 214-216. 